\title{
Thermal properties of oil palm nano filler/kenaf reinforced epoxy hybrid nanocomposites
}

\begin{abstract}
The aim of this research study was to fabricate nano oil palm empty fruit bunch (OPEFB)/kenaf/epoxy hybrid nanocomposites and to make comparative study on the thermal properties of nano OPEFB/kenaf/epoxy hybrid nanocomposites with the montmorillonite (MMT)/kenaf/epoxy hybrid nanocomposites and organically modified MMT (OMMT)/kenaf/epoxy hybrid nanocomposites. Epoxy based kenaf hybrid nanocomposites was prepared by dispersing the nano filler (nano OPEFB filler, MMT, OMMT) at 3\% loading through high speed mechanical stirrer followed by hand lay-up technique. Thermal properties of hybrid nanocomposites were analyzed through thermogravimetry analyzer (TGA), and differential scanning calorimetry (DSC). Obtained results specified that addition of nano OPEFB filler improves the thermal stability and char yield of kenaf/epoxy composites. Furthermore, the increase in decomposition temperature by the nano OPEFB filler was quite comparable to the MMT/kenaf/epoxy but relatively less than OMMT/kenaf/epoxy hybrid nanocomposites. We concluded from overall consequences that the nano OPEFB filler can be used as the promising and innovative alternative of existing expensive nano filler, with relatively lesser impact on the environment having marked pronounced impact on the construction, automotive, aerospace, electronics and semiconducting sectors as future industries based on bio-wastes with satisfactory light weight and thermal stability on other side.
\end{abstract}

Keyword: Nano OPEFB filler; Thermal stability; Kenaf; Epoxy hybrid nanocomposites 